\title{
Recent Results on Two-photon Physics at BABAR
}

\author{
V. P. Druzhinin* ${ }^{\dagger}$ \\ Budker Institute of Nuclear Physics, Novosibirsk, Russia \\ E-mail: druzhinin@inp.nsk.su
}

Recent BABAR results on two-photon processes are presented. A high statistics study of the twophoton production of the charmonium states $\eta_{c}$ and $\eta_{c}(2 S)$ is performed. The mass and width of $\eta_{c}$ and $\eta_{c}(2 S)$ are measured; the ratio of the decay probabilities to $K_{S} K^{+} \pi^{-}$and $K^{+} K^{-} \pi^{+} \pi^{-} \pi^{0}$ are determined. The latter mode is studied for the first time. The reactions $e^{+} e^{-} \rightarrow e^{+} e^{-} \gamma^{*} \gamma^{*} \rightarrow$ $e^{+} e^{-}+$pseudoscalar meson are studied in the single-tag mode for $\pi^{0}, \eta, \eta^{\prime}$, and $\eta_{c}$. From the measured differential cross sections the $Q^{2}$ dependencies of the photon-meson transition form factors are extracted. From these measurements we conclude that the pion distribution amplitude strongly differs from the distribution amplitudes of $\eta$ and $\eta^{\prime}$ mesons.

35th International Conference of High Energy Physics - ICHEP2010,

July 22-28, 2010

Paris, France

*Speaker.

${ }^{\dagger}$ On behalf of the BABAR Collaboration 


\section{Introduction}

Two-photon production of a resonance $R$ is studied at $e^{+} e^{-}$colliders in the process $e^{+} e^{-} \rightarrow$ $e^{+} e^{-} R$. The electrons in this process are scattered predominantly at small angles. For the pseudoscalar meson production, the effect of strong interactions is described by only one form factor $F\left(q_{1}^{2}, q_{2}^{2}\right)$ depending on the squared momentum transfers to the electrons.

Two-photon processes are usually studied in so called no-tag mode with both final electrons undetected. In this case the virtual photons emitted by electrons are practically real, the momentum transfers squared are close to zero. In no-tag mode the meson-photon transition form factor at zero $q^{2}$, s and the meson two-photon width are measured.

In the single tag-mode one of the final electrons is detected. The corresponding virtual photon is highly off-shell. From the measurement of the cross section richer information is extracted: the dependence of the meson form factor on $Q^{2}=-q_{1}^{2}$.

In this report we present results of no-tag and single-tag measurements performed with the BABAR detector at the PEP-II $e^{+} e^{-}$collider. The results are based on data with integrated luminosity of about $500 \mathrm{fb}^{-1}$ collected at the center-of-mass energy of $10.6 \mathrm{GeV}$.

No-tag two-photon events are selected by the requirement that the transverse momentum of detected hadron system is low. The single-tag events are selected with the detected and identified electron and with the fully reconstructed pseudoscalar meson, $\pi^{0}, \eta, \eta^{\prime}$, or $\eta_{c}$. It is required that the transverse momentum of the electron-plus-meson system be low and the missing mass in an event be close to zero.

\section{Measurement of $\eta_{c}$ and $\eta_{c}(2 S)$ parameters in the no-tag mode}

The $K_{S} K^{ \pm} \pi^{\mp}$ mass spectrum for no-tag events is shown in Fig. 1(a). The $\eta_{c}, J / \psi$, and $\eta_{c}(2 S)$ peaks are clearly seen over a non-resonant smooth background. The $J / \psi$ 's are produced in the initial state radiation process $e^{+} e^{-} \rightarrow J / \psi \gamma$. An evidence for the $\chi_{c 2} \rightarrow K_{S} K^{ \pm} \pi^{\mp}$ decay is also seen in Fig. 1b. From the fit to the mass spectrum the following $\eta_{c}$ parameters are determined [1]:

$$
\begin{aligned}
& m=2982.2 \pm 0.4 \pm 1.5 \mathrm{MeV} / c^{2}, \Gamma=31.7 \pm 1.2 \pm 0.8 \mathrm{MeV} \\
& \Gamma\left(\eta_{c} \rightarrow \gamma \gamma\right) B\left(\eta_{c} \rightarrow K \bar{K} \pi\right)=0.379 \pm 0.009 \pm 0.031 \mathrm{keV}
\end{aligned}
$$

These are the most precise measurements of the $\eta_{c}$ mass and width to date. The obtained value of $\Gamma\left(\eta_{c} \rightarrow \gamma \gamma\right) B\left(\eta_{c} \rightarrow K \bar{K} \pi\right)$ agrees with the CLEO measurement $0.407 \pm 0.022 \pm 0.028 \mathrm{keV}$ [2].

From the fit to the $K_{S} K^{ \pm} \pi^{\mp}$ mass spectrum in the vicinity of the $\eta_{c}(2 S)$ resonance the following values of the $\eta_{c}(2 S)$ mass and width are obtained:

$$
m=3638.3 \pm 1.5 \pm 0.6 \mathrm{MeV} / c^{2}, \Gamma=14.2 \pm 4.4 \pm 2.5 \mathrm{MeV}
$$

These results are preliminary. They are in reasonable agreement with the previous BABAR measurements [3]: $m=3630.8 \pm 3.4 \pm 1.0 \mathrm{MeV} / c^{2}$ and $\Gamma=17.0 \pm 8.3 \pm 2.5 \mathrm{MeV}$, obtained using 88 $\mathrm{fb}^{-1}$ data. The current PDG values for these parameters are $m=3637 \pm 4 \mathrm{MeV} / c^{2}$ and $\Gamma=14 \pm 7$ $\mathrm{MeV}$ [4]. The measured value of the $\eta_{c}(2 S)$ width is also in good agreement with an estimation based on a quark model: $\Gamma\left(\eta_{c}(2 S) \rightarrow g g\right) \approx \Gamma\left(\eta_{c}(1 S) \rightarrow g g\right) \Gamma(\psi(2 S) \rightarrow e e) / \Gamma(\psi(1 S) \rightarrow e e)=$ $12.1 \pm 1.0 \mathrm{MeV}$. 

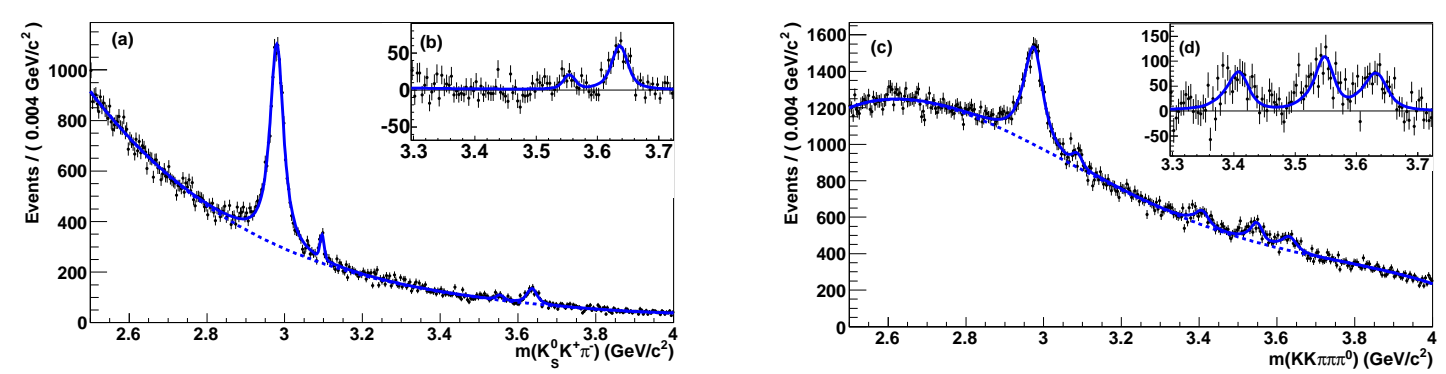

Figure 1: The $K_{S} K^{ \pm} \pi^{\mp}$ (a) and $K^{+} K^{-} \pi^{+} \pi^{-} \pi^{0}$ (c) mass spectra. The solid line is the fit result. The dashed line represents non-resonant background. The plots (b) and (d) show background subtracted spectra for the mass range $3.3-3.7 \mathrm{GeV} / \mathrm{c}^{2}$.

The mass spectrum for $K^{+} K^{-} \pi^{+} \pi^{-} \pi^{0}$ two-photon events is shown in Fig. 1(c). The signals from $\eta_{c}, \chi_{c 0}, \chi_{c 2}$, and $\eta_{c}(2 S)$ are seen. This is a first observation of the $K^{+} K^{-} \pi^{+} \pi^{-} \pi^{0}$ decay for these resonances. The $\eta_{c}(2 S)$ meson was previously observed in only $K_{S} K \pi$ decay mode. We have determined the ratios of the branching fractions into the two decay modes for $\eta_{c}$ and $\eta_{c}(2 S)$ :

$$
\begin{aligned}
& B\left(\eta_{c} \rightarrow K^{+} K^{-} \pi^{+} \pi^{-} \pi^{0}\right) / B\left(\eta_{c} \rightarrow K_{S} K^{ \pm} \pi^{\mp}\right)=1.42 \pm 0.06 \pm 0.26, \\
& B\left(\eta_{c}(2 S) \rightarrow K^{+} K^{-} \pi^{+} \pi^{-} \pi^{0}\right) / B\left(\eta_{c}(2 S) \rightarrow K_{S} K^{ \pm} \pi^{\mp}\right)=2.1 \pm 0.4 \pm 0.5 .
\end{aligned}
$$

These results are preliminary.

\section{Measurement of meson-photon transition form factors}

In perturbative $\mathrm{QCD}$, at large $Q^{2}$, the meson-photon transition form factor can be represented as a convolution of a calculable amplitude for $\gamma \gamma^{*} \rightarrow q \bar{q}$ with a nonperturbative meson distribution amplitude (DA) [5]. The latter describes the transition of the meson into two quarks.

Due to the relatively large $c$-quark mass, the $\eta_{c}$ form factor is rather insensitive to the shape of the $\eta_{c}$ distribution amplitude. Its $Q^{2}$ dependence is expected to be described by a monopole function with a pole parameter $\Lambda \sim 10 \mathrm{GeV}^{2}$ [6]. This value is close to the VDM prediction: $\Lambda=m_{J / \psi}^{2}=9.6 \mathrm{GeV}^{2}$.

The BABAR data on the $Q^{2}$ dependence of the normalized $\gamma \gamma^{*} \rightarrow \eta_{c}$ transition form factor [1] is fitted well by a monopole function. The found pole parameter $\Lambda=8.5 \pm 0.6 \pm 0.7 \mathrm{GeV}^{2}$ is in agreement with both VDM and QCD predictions, and with the result of the lattice QCD calculation: $\Lambda=8.4 \pm 0.4 \mathrm{GeV}^{2}[7]$.

For light pseudoscalars, the form factor depends strongly on the shape of the meson DA. Experimental data can be used to test different DA models. The BABAR results [8] on the scaled (multiplied by $Q^{2}$ ) $\gamma \gamma^{*} \rightarrow \pi^{0}$ transition form factor is shown in Fig. 2(a) together with CLEO and CELLO data $[9,10]$. The horizontal dashed line indicates the asymptotic limit for the scaled form factor $\left(Q^{2} F\left(Q^{2}\right)=\sqrt{2} f_{\pi} \approx 0.185 \mathrm{GeV}\right)$ predicted by pQCD [5]. The measured form factor exceeds the asymptotic limit at $Q^{2}>10 \mathrm{GeV}^{2}$. This means that the pion DA is significantly wider than the asymptotic DA. In particular, a very wide, flat DA was proposed in Refs. [11, 12, 13] to describe the $Q^{2}$ dependence of the pion form factor observed by BABAR. 

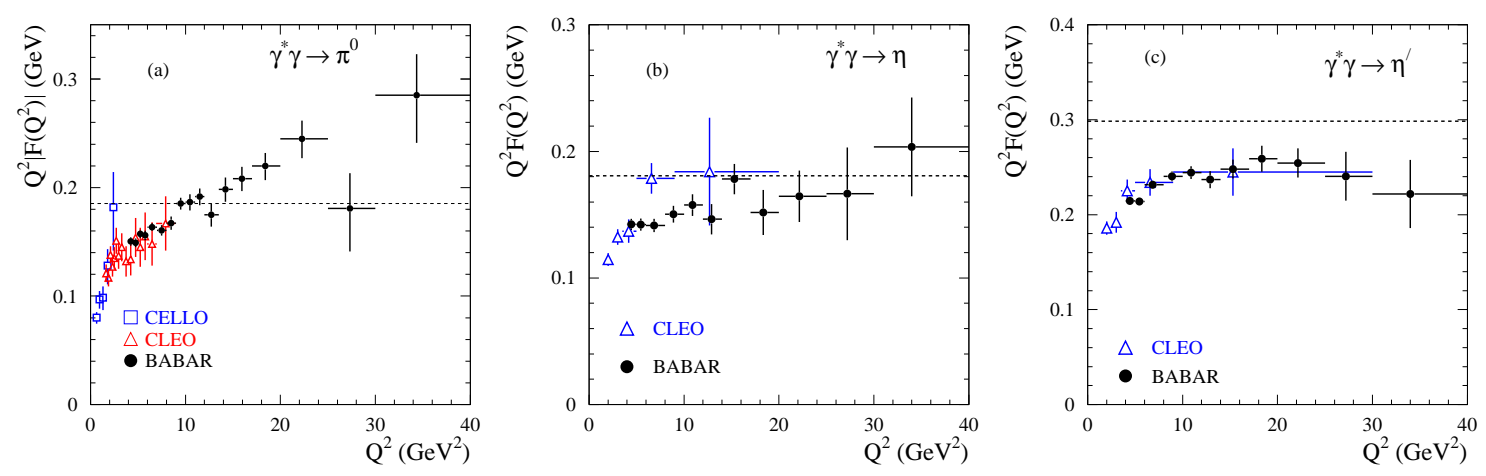

Figure 2: The scaled photon-meson transition form factors for $\pi^{0}$ (a), $\eta$ (b), and $\eta^{\prime}$ (c) mesons. The dashed lines indicate the asymptotic limits for the scaled form factors.

The BABAR preliminary results on the scaled $\gamma \gamma^{*} \rightarrow \eta$ and $\eta^{\prime}$ transition form factors measured in the $e^{+} e^{-} \rightarrow e^{+} e^{-} \eta^{(\prime)}$ reactions are shown in Figs. 2(b) and (c) in comparison with previous CLEO measurements [10]. We significantly improve the precision and extend the $Q^{2}$ region for form factor measurements. For $\eta^{\prime}$ our results and CLEO measurements are in good agreement. For $\eta$ the agreement is worse. The CLEO point at $7 \mathrm{GeV}^{2}$ lies higher than our data by about 3 sigmas. The dashed lines indicate the asymptotic limits for the scaled $\eta$ and $\eta^{\prime}$ form factors calculated in Ref. [14]. It is seen that $Q^{2}$ dependencies of the form factors for $\eta$ and $\eta^{\prime}$ differ from that for $\pi^{0}$. We conclude that BABAR results on the meson-photon transition form factors for light pseudoscalars indicate that the pion DA is significantly wider than the DA's of $\eta$ and $\eta^{\prime}$ mesons.

\section{References}

[1] J. P. Lees et al. [The BABAR Collaboration], Phys. Rev. D 81, 052010 (2010).

[2] D. M. Asner et al. [CLEO Collaboration], Phys. Rev. Lett. 92, 142001 (2004).

[3] B. Aubert et al. [BABAR Collaboration], Phys. Rev. Lett. 92, 142002 (2004).

[4] K. Nakamura et al. [Particle Data Group], J. Phys. G 37, 075021 (2010).

[5] G. P. Lepage and S. J. Brodsky, Phys. Rev. D 22, 2157 (1980).

[6] T. Feldmann and P. Kroll, Phys. Lett. B 413, 410 (1997).

[7] J. J. Dudek and R. G. Edwards, Phys. Rev. Lett. 97, 172001 (2006).

[8] B. Aubert et al. [The BABAR Collaboration], Phys. Rev. D 80, 052002 (2009).

[9] H. J. Behrend et al. [CELLO Collaboration], Z. Phys. C 49, 401 (1991).

[10] J. Gronberg et al. [CLEO Collaboration], Phys. Rev. D 57, 33 (1998).

[11] A. E. Dorokhov, arXiv:0905.4577 [hep-ph].

[12] A. V. Radyushkin, Phys. Rev. D 80, 094009 (2009).

[13] M. V. Polyakov, JETP Lett. 90, 228 (2009).

[14] T. Feldmann and P. Kroll, Phys. Rev. D 58, 057501 (1998). 